\title{
Epidural Abscess Related to Brucellar Spondylodiscitis Diagnosis by Polymerase Chain Reaction (PCR)
}

\author{
Mariana Costa* ${ }^{\circledR}$, Marina Vitorino ${ }^{\circledR}$, Beatriz Porteiro ${ }^{(0)}$, Whine Pedro ${ }^{(D}$, \\ Marinela Major ${ }^{\circledR}$, João Machado $\mathbb{}$ \\ Department of Internal Medicine, Hospital Professor Dr. Fernando da Fonseca EPE, Amadora, Portugal \\ Email: *mariana.oliv.costa@gmail.com
}

How to cite this paper: Costa, M., Vitorino, M., Porteiro, B., Pedro, W., Major, M. and Machado, J. (2019) Epidural Abscess Related to Brucellar Spondylodiscitis Diagnosis by Polymerase Chain Reaction (PCR). Advances in Infectious Diseases, 9, 238-242.

https://doi.org/10.4236/aid.2019.93017

Received: June 28, 2019

Accepted: August 26, 2019

Published: August 29, 2019

Copyright $\odot 2019$ by author(s) and Scientific Research Publishing Inc. This work is licensed under the Creative Commons Attribution International License (CC BY 4.0).

http://creativecommons.org/licenses/by/4.0/

(c) (i) Open Access

\begin{abstract}
The localized forms of brucellosis, particularly osteoarticular, by their symptomatology usually nonspecific, still continue to be a diagnosis challenge, being fundamental to raise a high degree of suspicion based on a careful epidemiological history. The authors describe the case of a 69 year old farmer that was admitted due to an insidious lower back pain with irradiation to the left lower limb accompanied by nocturnal sweating, anorexia and weight loss. The imaging studies revealed a spondylodiscitis in L3-L4 with an associated epidural abscess. The diagnosis of brucellosis was brought on by the occupational exposure to sheep and was confirmed by a positive PCR to Brucella in the product gathered by aspiration from the epidural abscess and also by the serology (ELISA and Rose Bengal). Despite the initial therapy with doxycycline, rifampicin and gentamycin (the last one for 1 week), the patient had a clinical and imagiologic worsening, determining surgical decompression. After the surgery, antiobiotherapy with doxycyline and rifampicin was maintained and a cycle of gentamicin was started, this time for a month, with a favorable clinical evolution.
\end{abstract}

\section{Keywords}

Lower Back Pain, Spondylodiscitis, Brucellosis, PCR

\section{Introduction}

Despite all the progress in the eradication of brucellosis in animals, the disease is still prevalent in humans due to high risk procedures as direct contact with infected animals and their secretions, contaminated aerosol inhalation and con- 
sumption of unpasteurized milk products [1] [2] [3].

Spinal involvement is one of the most frequent focal complications of brucellosis, generally in the form of sacroiliitis in young patients and spondylodiscitis of the lumbar column in the elderly. Vertebral osteomyelitis is a severe complication associated with a paravertebral, epidural or psoas abscess [2] [4].

In the acute and systemic phases of the disease, the diagnosis of brucellosis is made by the positive results in blood cultures. In the localized forms, the diagnosis is often made by serology, however, in the recent years, PCR methods are being used more frequently [2] [4] [5].

\section{Case Report}

A 69 year old farmer was admitted to our emergency department in $24^{\text {th }}$ of October due to a clinical condition that had last for the past 3 months, characterized by lower back pain that irradiated to the left lower limb and that didn't get better with rest. At the same time, he reported having nocturnal sweating without any characteristic smell, a weight loss quantified in $5 \mathrm{Kg}$ and anorexia. He denied having fever or chills.

Due to these complaints, he had been observed previously in another hospital where a CT scan of lumbar column was made, revealing degenerative alterations, so he was discharged medicated with pain killers.

From the past epidemiological history, it was pointed out the fact that he had a farm and was frequently in contact with animals namely, goats and sheep. The patient recalled that 4 months before the beginning of the symptoms, he had helped in the delivery of a sheep, without protection, whose fetuses presented with several malformations. Nevertheless, he denied consumption of unpasteurized milk and milk products.

On the examination the temperature was $36^{\circ}$, the blood pressure 102/65 $\mathrm{mmHg}$ and the heart rate 77 beats per minute. The cardiopulmonary auscultation was normal; there was no hepatosplenomegaly. There was no focal tenderness in the lumbar spine. Lasègue signal was present at left.

In the laboratory, evaluation stood out: $\mathrm{Hb} 13.5 \mathrm{~g} / \mathrm{dL}$, Leucocytes 6100, Platelets 387,000, AST 8 U/L; ALT 42 U/L; FA 136.19 U/L; GGT 95 U/L; LDH 111 $\mathrm{U} / \mathrm{L}$; B. total $0.35 \mathrm{mg} / \mathrm{dL}$, creatinine $0.85 \mathrm{mg} / \mathrm{dL}$, urea $45.1 \mathrm{mg} / \mathrm{dL}, \mathrm{PCR} 1.78 \mathrm{mg} / \mathrm{dL}$, VS $38 \mathrm{~mm}$ in the first hour.

The chest X-ray had no alterations.

An MR of the lumbar column was made (Figure 1). In the portion next to the body of L3 and L4, there was an alteration of the signal emission after the administration of gadolinium. There was also an anterior epidural lesion of L4 that was continuous with posterior portion of the intervertebral disk of L3-L4 that conditioned molding of the adjacent thecal sac.

Concerning the imagiologic aspects of the $\mathrm{MR}$, a CT scan guided percutaneous biopsy of the epidural collection was made, in which, the PCR for Brucella species was positive (PCR kit AmpliSens Brucella spp.-FRT, produced by $E$ coli 


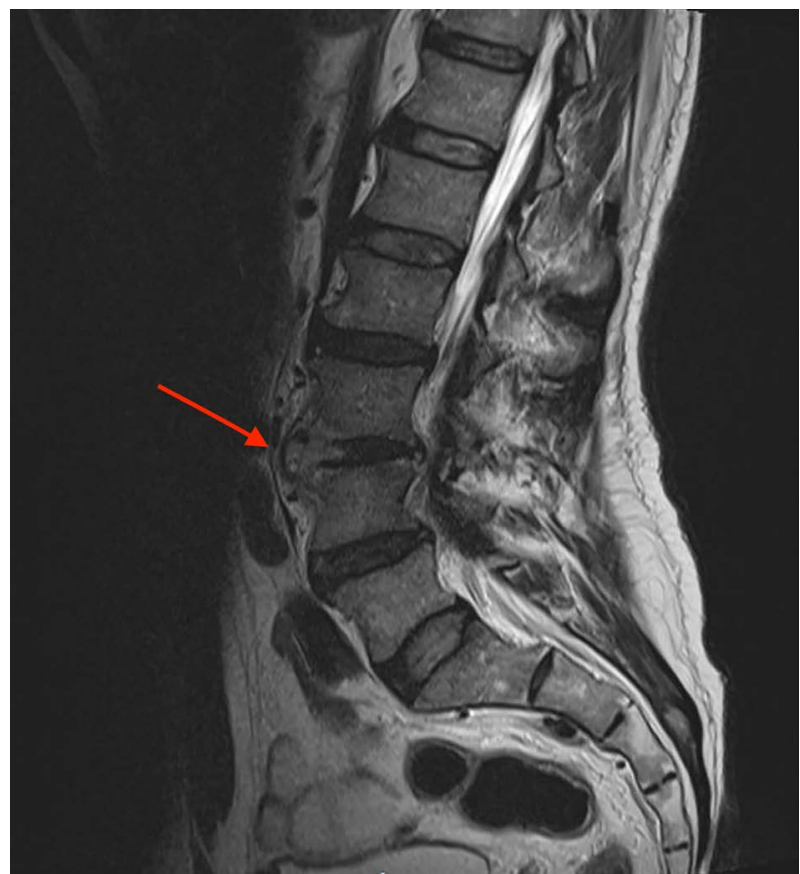

Figure 1. MR at the admission on the service.

s.r.o.). The corresponding culture was negative and the serology by ELISA IgG and IgM and the Rose of Bengal, were positive.

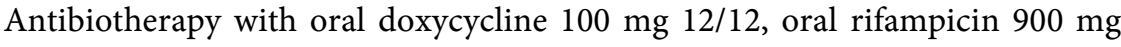
once daily and intravenous gentamicin $180 \mathrm{mg}$ once daily, the last one for 7 days, was initiated. The patient had a favorable evolution and was discharged with the indication to continue the antibiotherapy with doxycycline and rifampicin.

Two weeks after the discharge, the patient came again to the hospital, with back pain irradiating to the left leg. A new MR was made showing an expansion the epidural infectious component in its antero-superior extension that caused compression in the adjacent thecal sac with total deletion of the subarachnoid space (Figure 2).

In face of the clinical and imagiologic worsening the patient was referred to Neurosurgery where he was submitted to surgery where a laminectomy of L3-L4, foraminectomy of L3-L4 and L4-L5, and decompression of the thecal sac, were made. The cultural exams of the material collected during surgery, were negative.

After the surgery the antibiotherapy with doxycycline and rifampicin was continued and another cycle of gentamicin was made, this time for 1 month.

The patient had a significant clinical improvement without pain and was able to walk without help. The MR at the fourth day after the surgery showed adequate decompression of the nerves of the cauda equine and reduction of the dimensions of the abscess.

The patient was discharged medicated with doxycycline and rifampicin for 6 months.

At the $9^{\text {th }}$ month re-evaluation, the patient had no complaints and the MR showed almost complete resolution of the inflammatory process. 


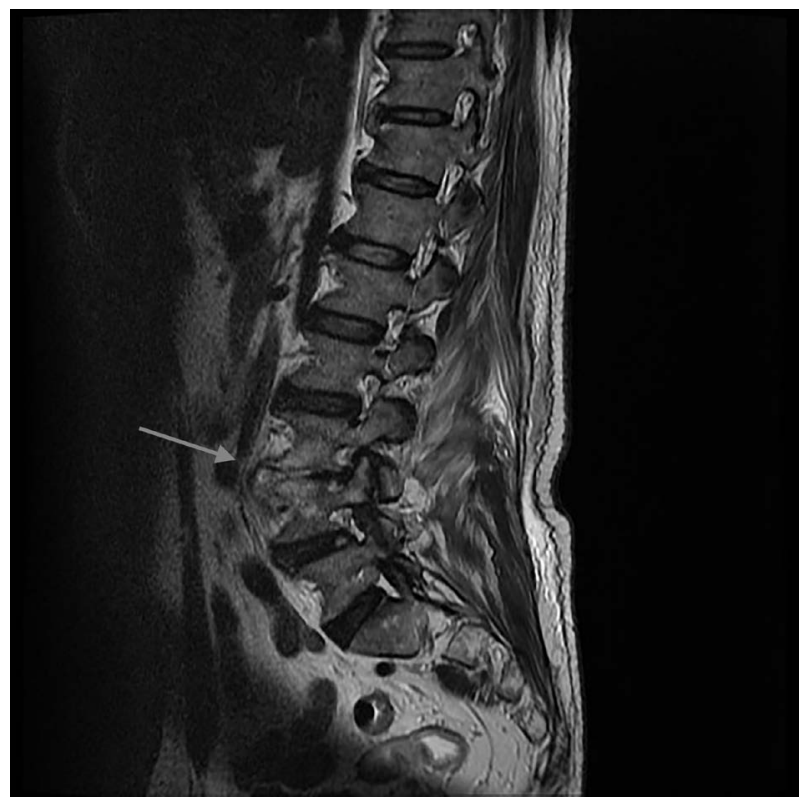

Figure 2. MR 2 weeks after the hospital discharge.

\section{Discussion}

The focal forms of brucellosis in spine are a diagnostic challenge due to their subtle expression as back pain that can be confused with another diagnosis. Our patient presented with a three month back pain with inflammatory characteristics, without fever. He was observed before in another hospital where a CT scan was made revealing degenerative alterations.

The MR was extremely important in the diagnosis because it was able to identify the abscess associated with a spondylodiscitis in L3-L4.

In the face of the imagiologic findings, it was the epidemiological history that pointed towards the diagnosis of brucellosis due to the delivery of a sheep whose fetuses presented with several malformations, aspect compatible with active infection due to Brucella.

As it would be expected, outside the acute phase of the infection, in our case the blood cultures were negative. The diagnosis was firmly established by the positivity of the PCR in the aspired abscess identified in the MR and simultaneously by serology (ELISA and Rose of Bengal).

The positivity of the PCR displays the crescent expansion of the molecular biology in the diagnosis of brucellosis. It has been increasingly used in peripheral blood and other samples, showing a high sensibility and specificity in patients with various stages of the disease-bacteremia and focal complications [4] [6].

In the treatment of spondylodiscitis to Brucella the combination of doxycycline and an aminoglycoside, which is frequently gentamicin, associated in some series with rifampicin, has recognized effectiveness [3] [7].

In our case, the treatment consisted of 7 days of gentamicin, as recommended by the literature, associated with rifampicin and doxycycline, with an apparent response in the beginning. However, the patient got worst, both from the clinical 
and imagiologic point of view in the fourth week that determined a surgical intervention.

\section{Conclusions}

Our case recalls a focal presentation of brucellosis in which the diagnosis was established due to the positivity of the PCR in the epidural abscess and positive serology.

The case illustrates the importance that PCR methods have in the diagnosis of focal forms of brucellosis, and at the same time, reminds of the relevance of a careful epidemiological history.

At last, this case reinforces the discussion about the importance of the inclusion of an aminoglycoside in the initial therapeutic scheme, namely if a prolonged administration would had avoided the surgical intervention.

\section{Conflicts of Interest}

The authors declare no conflicts of interest regarding the publication of this paper.

\section{References}

[1] Pappas, G., Akritidis, N., Bosilkovski, M., et al. (2005) Brucellosis. The New England Journal of Medicine, 352, 2325-2336. https://doi.org/10.1056/NEJMra050570

[2] Doganay, M. and Aygen, B. (2003) Human Brucellosis: An Overview. International Journal of Infectious Diseases, 7, 173-182. https://doi.org/10.1016/S1201-9712(03)90049-X

[3] Mandell, Douglas and Bennett's (2015) Principles and Practice of Infectious Diseases. 8th Edition, Elsevier Saunders, Philadelphia, 2584-2589.

[4] Ulu-Kilic, A., Karakas, A., Erdem, H., et al. (2014) Update on Treatment Options for Spinal Brucellosis. Clinical Microbiology and Infection, 20, 75-82. https://doi.org/10.1111/1469-0691.12351

[5] Shehabi, A., Shakir, K., El-Khaterb, M., et al. (1990) Diagnosis and Treatment of 106 Cases of Human Brucellosis. Journal of Infection, 20, 5-10. https://doi.org/10.1016/S0163-4453(90)92214-6

[6] Navarro, E., Caso, M.A. and Solera, J. (2004) Diagnosis of Human Brucellosis Using PCR. Expert Review of Molecular Diagnostics, 4, 115-123. https://doi.org/10.1586/14737159.4.1.115

[7] Buzgan, T., Karahocagil, M.K., Irmak, H., et al. (2010) Clinical Manifestations and Complications in 1028 Cases of Brucellosis: A Retrospective Evaluation and Review of Literature. International Journal of Infectious Diseases, 14, e469-e478. https://doi.org/10.1016/j.ijid.2009.06.031 